\title{
Decentralized SLAM for Pedestrians without direct Communication
}

\author{
Alexander Kleiner and D. Sun
}

\section{Post Print}

N.B.: When citing this work, cite the original article.

(C2007 IEEE. Personal use of this material is permitted. However, permission to reprint/republish this material for advertising or promotional purposes or for creating new collective works for resale or redistribution to servers or lists, or to reuse any copyrighted component of this work in other works must be obtained from the IEEE.

Alexander Kleiner and D. Sun, Decentralized SLAM for Pedestrians without direct Communication, 2007, In Proc. of the IEEE/RSJ Int. Conf. on Intelligent Robots and Systems (IROS), 1461-1466.

http://dx.doi.org/10.1109/IROS.2007.4399013

Postprint available at: Linköping University Electronic Press

http://urn.kb.se/resolve?urn=urn:nbn:se:liu:diva-72565 


\title{
Decentralized SLAM for Pedestrians without direct Communication
}

\author{
Alexander Kleiner and Dali Sun \\ Institut für Informatik \\ University of Freiburg \\ 79110 Freiburg, Germany \\ \{kleiner,sun\}@informatik.uni-freiburg.de
}

\begin{abstract}
We consider the problem of Decentralized Simultaneous Localization And Mapping (DSLAM) for pedestrians in the context of Urban Search And Rescue (USAR). In this context, DSLAM is a challenging task. First, data exchange fails due to cut off communication links. Second, loop-closure is cumbersome due to the fact that firefighters will intentionally try to avoid performing loops when facing the reality of emergency response, e.g. while they are searching for victims.

In this paper, we introduce a solution to this problem based on the non-selfish sharing of information between pedestrians for loop-closure. We introduce a novel DSLAM method which is based on data exchange and association via RFID technology, not requiring any radio communication. The approach has been evaluated in both semi-indoor and outdoor environments. The presented results show that sharing information between single pedestrians allows to optimize globally their individual paths, even if they are not able to communicate directly.
\end{abstract}

\section{INTRODUCTION}

Pedestrian navigation and localization is a growing field motivated from the context of Location Based Services (LBS) [13], navigation for the Blind [11], and emergency responder tracking [16]. Particularly in the field of emergency response, the efficiency of rescue teams, e.g. when performing the search for victims after a disaster, depends on their ability to coordinate and thus to be aware of their locations. However, to be aware of locations during exploration within collapsed buildings is a challenging task. In urban environments GNSS (Global Navigation Satellite System) positioning is affected by the multipath propagation problem [5]. Buildings in the vicinity of the receiver reflect GNSS signals, resulting in secondary path propagations with longer propagation time, causing erroneous position estimates. Furthermore, communication might not be possible due to structures made of reinforced concrete, and the ability to re-observe landmarks might be affected by limited visibility due to smoke and fire.

One solution to this problem is to equip firefighters with assistance systems, such as wearable devices [9],

\footnotetext{
${ }^{1}$ This research was partially supported by DFG as part of the collaborative research center SFB/TR-8 Spatial Cognition
}

[16], performing Simultaneous Localization And Mapping (SLAM) without cognitive load, e.g. without requiring interactions by the user. SLAM methods work with the principle of map improvement through loop-closure, i.e. to improve the map globally each time places have been re-observed. However, when facing the reality of emergency response, firefighters will try to intentionally avoid performing loops, e.g. while they are searching for victims.

In this paper, we introduce a solution to this problem based on non-selfish information sharing between pedestrians via the deployment of RFID technology. Hereby the pose of each pedestrian is automatically tracked by a PDR (Pedestrian Dead Reckoning) method, which recognizes human footsteps analytically from acceleration patterns. We introduce a centralized approach that improves the joint network of all trajectories by minimizing the Mahalanobis distance [14], while utilizing RFID transponders for data association [10]. Then, we extend this method towards a novel method for Decentralized SLAM (DSLAM) that allows to globally optimize the network in a decentralized manner by exchanging information via the memory of RFIDs. Hence, the method does not depend on direct radio communication.

RFID technology offers many advantages in harsh environments: First, RFIDs can be operational up to temperatures of $450{ }^{\circ} \mathrm{C}$ [2]. Second, their size can be below a half millimeter, e.g. the $\mu$-Chip from Hitachi, making it possible to deploy them in masses in disaster areas, for example, distributed by UAVs or UGVs [10]. Third, there memory capacity increases continuously, reaching currently more than $4 \mathrm{MBit}$ [15]. We conducted extensive experiments in an urban outdoor environment. The presented results show that the introduced method allows to improve single trajectories significantly by exchanging data between the agents.

The tracking of human motion has mainly been studied based on the analysis of acceleration patterns [3], [12], [6]. Furthermore, infrastructure-based localization has been studied, e.g. based on WLAN [4], and super-distributed RFID tag infrastructures [1], and also specifically in the context of emergency response [7], [16]. However, these methods are mainly designed from a single agent perspective, i.e. do not exploit the potential advantage 
of data sharing.

Efficient solutions for decentralized SLAM have been introduced in the past [18], [17], [8]. These approaches assume that at least low-bandwidth radio communication between the agents is possible. They solve the double counting problem, i.e. the multiple propagation of equal information via different paths, by probabilistic methods, such as Bounded Covariance Inflation (BCI) [18], and Covariance Intersection (CI) [17]. We show that this problem can be solved also by managing estimates locally within the memory of RFIDs.

\section{Pedestrian Dead Reckoning (PDR)}

We denote the two-dimensional pose of pedestrians by the vector $l=(x, y, \theta)^{T}$. In order to represent uncertainties, the pose is modeled by a Gaussian distribution $N\left(\mu_{l}, \Sigma_{l}\right)$, where $\mu_{l}$ is the mean and $\Sigma_{l}$ a $3 \times 3$ covariance matrix. Motion estimation of human walking yields the traveled distance $d$ and angle $\alpha$, likewise modeled by a Gaussian distribution $N\left(u, \Sigma_{u}\right)$, where $u=(d, \alpha)$ and $\Sigma_{u}$ is a $2 \times 2$ covariance matrix expressing estimation errors. The pose at time $t$ can be updated from input $u_{t}$ as follows:

$$
\begin{array}{r}
l_{t}=F\left(l_{t-1}, u_{t}\right)=\left(\begin{array}{c}
x_{t-1}+\cos \left(\theta_{t-1}\right) d_{t} \\
y_{t-1}+\sin \left(\theta_{t-1}\right) d_{t} \\
\theta_{t-1}+\alpha_{t}
\end{array}\right), \\
\Sigma_{l_{t}}=\nabla F_{l} \Sigma_{l_{t-1}} \nabla F_{l}^{T}+\nabla F_{u} \Sigma_{u} \nabla F_{u}^{T}, \\
\text { where } \Sigma_{u}=\left(\begin{array}{cc}
d \sigma_{d}^{2} & 0 \\
0 & \alpha \sigma_{\alpha}^{2}
\end{array}\right)
\end{array}
$$

and $\nabla F_{l}$ and $\nabla F_{u}$ are partial matrices of the Jacobian matrix $\nabla F_{l u}$.

Input $u_{t}$ is acquired from human walking after a method that has been introduced by Ladetto et al. [12] Human walking generates a vertical acceleration with a maximum value if a foot is placed on the ground. By detecting these maxima in the vertical acceleration curve it is possible to detect and count the occurrence of footsteps. The distance estimate is coupled with an IMU (Inertial Measurement Unit), yielding the walking direction $\theta$.

\section{CENTRALIZED RFID-SLAM}

In centralized RFID-SLAM we assume that pedestrians (e.g. firefighters) are able to fully communicate with a central server (e.g. a command post). Furthermore, they track their pose by the PDR method described in Section II, while actively distributing and observing RFID tags in the environment. We assume that RFID detections $^{1}$ are within a range of $<1$ meter, which allows to cover corridors and doorways, while providing sufficient positioning accuracy. In the following, we will use the term "field agent", referring to the software agent executed on the pedestrian's wearable computer.

\footnotetext{
${ }^{1}$ RFID detection means basically the reading of an unique ID without any position information.
}

Each time an RFID has been observed by a field agent, a message is generated and send to a central server. The message contains the ID of the previously visited RFID $i$ and currently visited RFID $j$, as well as an estimate of the local displacement $\hat{d}_{i j}=d_{i j}+\Delta d_{i j}$ between both RFIDs estimated by Equation 1. The local displacement $\hat{d}_{i j}$ is defined by the vector $\left(\Delta \hat{x}_{i j}, \Delta \hat{y}_{i j}, \Delta \hat{\theta}_{i j}\right)^{T}$, where $\Delta \hat{x}_{i j}$ and $\Delta \hat{y}_{i j}$ denote the relative spacial displacement, and $\Delta \hat{\theta}_{i j}$ the relative orientation change. Our goal is to compute a globally consistent map, i.e. to determine the true locations of the RFIDs from all observations communicated to the station. This map can then be communicated back to the field agents for improving their individual pose estimates.

We denote the true pose vectors of $n+1$ RFID nodes by $l_{0}, l_{1}, \ldots, l_{n}$, and the function calculating the true displacement $\left(\Delta x_{i j}, \Delta y_{i j}, \Delta \theta_{i j}\right)^{T}$ between a pair of nodes $\left(l_{i}, l_{j}\right)$ is denoted as measurement function $d_{i j}$. The central station incrementally builds a global graph from all displacement estimates communicated by the field agents through utilizing the unique ID of RFIDs for data association. The constructed graph $G=(V, E)$ consists of vertices $V$ and edges $E$, where each vertex represents an RFID tag, and each edge $\left(V_{i}, V_{j}\right) \in E$ represents an estimate $\hat{d}_{i j}$ with covariance matrix $\Sigma_{i j}$ between two RFID tags associated with vertices $V_{i}$ and $V_{j}$, respectively. The subgraphs from all field agents are unified in the following way: On the one hand, if the same vertex has been observed twice, a loop has been detected in the graph. A detected loop is modeled by a pseudo edge between the same RFID node with distance $\hat{d}_{i i}$ set to $(0,0, \Delta \theta)^{T}$, where $\Delta \theta$ denotes the angle difference between the two pose estimates of the RFID. Furthermore, under the assumption that RFIDs are detected at $95 \%$ probability if they are within maximal reading range $d_{M}$, the according covariance matrix is modeled by:

$$
\Sigma_{i i}=\left(\begin{array}{ccc}
2^{2} d_{M}^{2} & 0 & 0 \\
0 & 2^{2} d_{M}^{2} & 0 \\
0 & 0 & \sigma_{\theta}^{2}
\end{array}\right),
$$

where $\sigma_{\theta}^{2}$ is the linearized variance of the angle, and $2^{2} d_{M}^{2}$ the variance of the normal distribution over the interval $\left[-2 d_{M} ; 2 d_{M}\right]$. On the other hand, if two or more field agents observe the same edge, i.e. their trajectory overlaps between two or more neighboring RFIDs, both observations are merged by an extended Kalman filter.

Finally, the constructed graph, and thus the underlying map represented by RFID locations is globally corrected by minimizing the Mahalanobis distance [14]. Here, the goal is to find the true locations of the $l_{i j}$ given the set of measurements $\hat{d}_{i j}$ and covariance matrices $\Sigma_{i j}$. This can be achieved after the maximum likelihood concept by the following minimization:

$$
l=\underset{l}{\arg \min } \sum_{i, j}\left(d_{i j}-\hat{d}_{i j}\right)^{T} \Sigma_{i j}^{-1}\left(d_{i j}-\hat{d}_{i j}\right),
$$


where $l$ denotes the concatenation of poses $l_{0}, l_{1}, \ldots, l_{n}$. Since measurements are taken relatively, it is assumed without loss of generality that $l_{0}=0$ and $l_{1}, \cdots, l_{n}$ are relative to $l_{0}$. Moreover, the graph is considered as fully connected, and if there does not exist a measurement between two nodes, the inverse covariance matrix $\Sigma_{i j}^{-1}$ is set to zero. In order to solve the optimization problem analytically, Equation 5 can be transformed into matrix form For sake of simplicity we firstly assume that $d_{i j}=l_{i}-l_{j}$, i.e. displacements do not contain angle components. Then, the measurement functions for all edges can be described by the following matrix equation:

$$
d=h l
$$

where vector $l$ denotes the concatenation of pose vectors $l_{0}, \ldots, l_{n}$ with the dimension $n d$, vector $d$ denotes the concatenation of all pose differences $d_{i j}=l_{i}-l_{j}$, and matrix $h$ is a index matrix with elements $\{-1,0,1\}$. Consequently, Equation 5 can be rewritten in matrix form by

$$
l=\underset{l}{\arg \min }(\hat{d}-h l)^{T} \Sigma_{\hat{d}}^{-1}(\hat{d}-h l),
$$

where $h l$ denotes the measurement function as described by Equation $6, \hat{d}$ denotes the concatenation of observations $\hat{d}_{i j}$, and $\Sigma_{\hat{d}}^{-1}$ denotes the inverse covariance matrix of $\hat{d}$, consisting of the inverse sub-matrices $\Sigma_{i j}$. Finally, the minimization problem can be solved by

$$
l=\left(h^{T} \Sigma_{\hat{d}}^{-1} h\right)^{-1} h^{T} \Sigma_{\hat{d}}^{-1} \hat{d}
$$

and the covariance of $l$ can be calculated by

$$
\Sigma_{l}=\left(h^{T} \Sigma_{\hat{d}}^{-1} h\right)^{-1} .
$$

Note that equation 5 has to be linearized by a Taylor expansion if poses contain angles [14], which will be omitted here due to space constraints.

Since the computation of the solution requires a matrix inversion, the network can be optimized in $O\left(n^{3}\right)$, where $n$ denotes the number of RFIDs. However, this computation can be performed much more efficiently in practice since the matrix that has to be inverted is a sparse matrix, consisting of covariances from all pose estimates obtained between the nodes. This is due to the fact that when applying the method in practice, only a little amount of nodes in the graph are connected with each other and hence most covariances $\Sigma_{i j}$ are set to zero.

\section{DECENTRALIZED RFID-SLAM (DRFID-SLAM)}

In emergency response scenarios, communication links between agents or to a central station might be cutoff temporarily or constantly. One obvious reason is that within building structures radio waves might not be able to penetrate walls made of reinforced concrete. Otherwise, it might also be likely that first responders, which search for victims on a large terrain, are simply out of communication range. Moreover, they might visit the same locations at different times, and thus also not be able to communicate. Therefore, we assume in Decentralized RIFD-SLAM (DRFID-SLAM) that radio communication is constantly perturbed. Note that this assumption is hard, compared to other approaches that require at least low-bandwidth communication channels between the agents [18], [17], [8]. Furthermore, we assume that RFIDs have sufficient memory for storing RFID graph structures as described in Section III.

The basic idea of DRFID-SLAM is to utilize the memory of RFID tags for learning the topology of the surrounding RFID graph. When agents pass a tag, they update their knowledge on the graph topology by reading subgraph data from the tag, as well as synchronize the tag by writing subgraph data they have collected. By this, agents are able to learn a subgraph larger than their own trajectory, i.e. extended by trajectories of other agents. The union of trajectories might lead to subgraphs containing loops, facilitating the correction of single agent trajectories even they are loop-less.

The procedure can be considered as a step-wise information propagation through the network of RFIDs, where each visited RFID learns about nearby RFID nodes from the trajectory of its visitor, as well as from trajectories that were previously stored by other agents in the nodes passed by the visitor. Therefore, after a sufficient amount of trajectories has been traveled, a single RFID node memorizes the whole infrastructure of the network. Note that for supporting map improvements, RFIDs do not necessarily have to store the whole network structure.

One problem arising in the context of distributed SLAM is the double counting of information propagated via different paths, i.e. rumor propagation. While this problem typically requires to apply probabilistic methods, such as Bounded Covariance Inflation (BCI) [18], and Covariance Intersection (CI) [17], it can be solved rather trivially with RFIDs. Here the basic idea is to manage displacement estimates locally in the memory of RFIDs, i.e. to distinguish each estimate by location and time where and when it has been observed.

We distinguish edges as managed and propagated in the memory of RFIDs and introduce a counter $C_{i}$ attached to each RFID, which is locally updated each time an agent writes information concerning a managed edge. Edges that are managed by node $i$ are all the adjacent edges that have $i$ in common, i.e. those edges that directly connect to $i$. Consider the case that an agent travels from RFID $i$ to RFID $j$ and generates thereby a new estimate $\left(\hat{d}_{i j}, \Sigma_{i j}\right)$. Then, RFID $j$ is the managing node, i.e. manages the edge between $i$ and $j$. Consequently, the agent stores in RFID $j$ a new entry $e_{i j}=\left\langle i, j, \hat{d}_{i j}, \Sigma_{i j}, C_{j}\right\rangle$, consisting of the IDs of the two visited RFIDs $i$ and $j$, their displacement estimate, and the current count value of the RFID, which is 
incremented after writing. Obviously, there can be two nodes managing the same edge since agents can either travel from $i$ to $j$ or from $j$ to $i$. Therefore, we have to distinguish both cases during propagation, which is carried out by defining that $\langle i, j, \cdots\rangle \neq\langle j, i, \cdots\rangle$, i.e. the managing node corresponds to the second node in the notation.

Propagated edges are managed edges after they have been copied by agents. When visiting a node, the agent performs a union operation between all entries stored in the memory of the RFID, denoted by subgraph $R_{i}$, and its own subgraph, denoted by $A_{i}$, and propagates them further to other RFIDs. The double counting of identical estimates is prevented by ensuring that there always exists only one unique entry for each combination of $i, j$, and $C_{j}$ and unique sequence $\langle i, j\rangle$ within each RFID, where $C_{j}$ is the counter value of RFID $j$ during the creation of the managed edge. The complete procedure for updating RFID nodes is shown by Algorithm 1. Note that the agent updates its own subgraph $A_{i}$ by the same procedure, while considering propagated edges only.

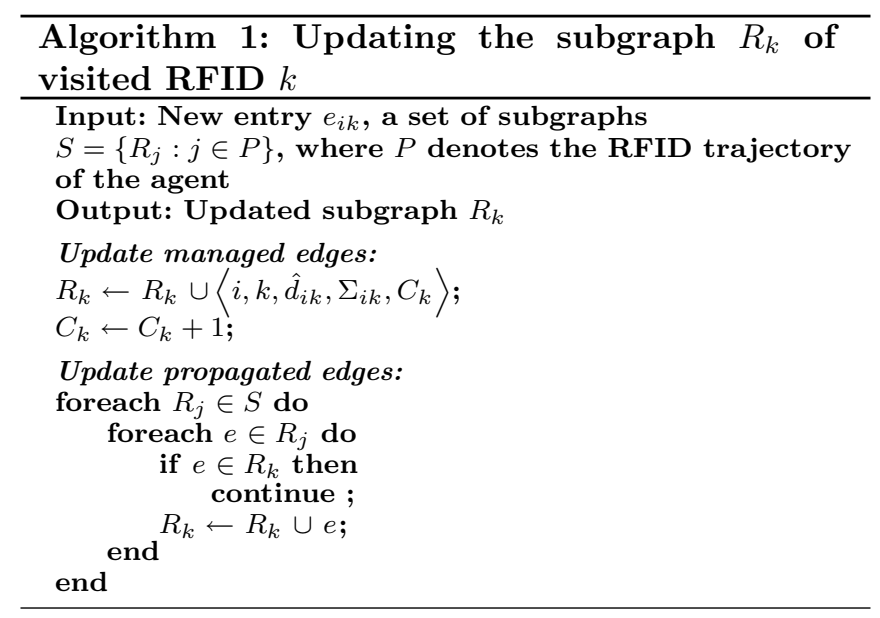

Finally, field agents are able to improve their pose estimate locally by correcting their trajectories unified with graph structures learned form the other agents.

\section{EXPERIMENTAL RESULTS}

We conducted extensive experiments in urban scenarios, while obtaining ground truth data from a GNSS device for each RFID location. For this purpose, we utilized the GPSlim236 GPS receiver from Holux, which is equipped with SiRF Star III technology. The receiver allows to track up to 20 satellites at an update rate of $1 \mathrm{~Hz}$ and has a position accuracy of $5-25$ meters. Furthermore, the receiver is able to process Differential GPS (DGPS) data from the EGNOS system, improving the horizontal position accuracy towards $<2.2$ meters and vertical position accuracy towards $<5$ meters at $95 \%$ of the time.

For the purpose of pedestrian dead-reckoning we utilized the MTi IMU from Xsens, which combines a tri- axial accelerometer and a tri-axial gyroscope with a triaxial magnetometer. Due to the simultaneous integration of gyro and compass data, the device provides a drift-free orientation vector that is stable towards minor perturbations caused by external magnetic sources. The device was attached to a test person equipped with a wearable computer for measuring acceleration patterns, as well as the azimuth orientation. Based on an empirical evaluation, pose tracking uncertainty was modeled with $\sigma_{\hat{d}}^{2}=(0.05 m / m)^{2} d$, and $\sigma_{\hat{\theta}}^{2}=\left(15^{\circ}\right)^{2}$.

Without loss of generality we simulated the RFID sensor by manually marking places with numbered labels that are re-observable by the test person. Each time the test person deployed a new label, or was within range of a label that has been deployed before, the RFID observation has been logged to a data base, with each entry of the data base consisting of a time stamp and the identification number of the observed RFID. During the experiments, re-observations took place within a maximal distance of 1 meter. Hence, the covariance matrix in Equation 4 was modeled with $d_{M}=1 \mathrm{~m}$.

\section{A. Semi-Indoor Experiment}

The semi-indoor experiment was carried out on the campus of the University of Freiburg, including many accessible buildings which have been entered by the test person. We measured that some of these buildings contain magnetic fields disturbing the angle estimate of the PDR method, as for example, metal stairs or metal doors. Figure 1 provides an overview of the area, generated with GoogleEarth. During this experiment, the test person traveled six trajectories with different starting and ending locations, while performing pose tracking with the PDR method previously described, and while distributing and re-observing RFIDs. In order to

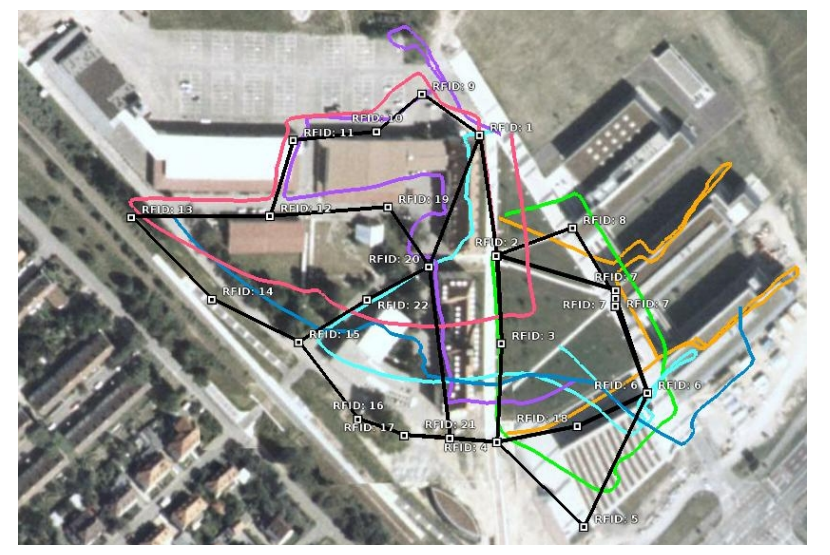

Fig. 1. Result from the semi-indoor experiment: Each colored line indicates the pose tracking of a single pedestrian. Black lines and squares show the jointly corrected graph of RFIDs.

visualize the PDR trajectories, we utilized the accurate starting locations taken from the ground truth data and projected each PDR trajectory with respect to its starting location on the map. In Figure 1 each trajectory 
is shown with a different color. As can be seen, position accuracy decreases with increasing length of the traveled trajectory.

All trajectories have been collected and merged into a single graph for applying the centralized method described in Section III. The corrected locations of RFIDs are shown by the small black squares in Figure 1. Furthermore, we computed the average Cartesian error of estimated RFID locations with respect to ground truth. This has been carried out by aligning each estimated trajectory to its global starting position and then computing the Cartesian error of each visited RFID. Figure 2 depicts the averaged errors according to each pedestrian. It shows the uncorrected trajectory, the single trajectory corrected on its own, and the trajectory corrected from the unified graph. As can be seen, the correction based on the unified graph yields best results for all pedestrians.

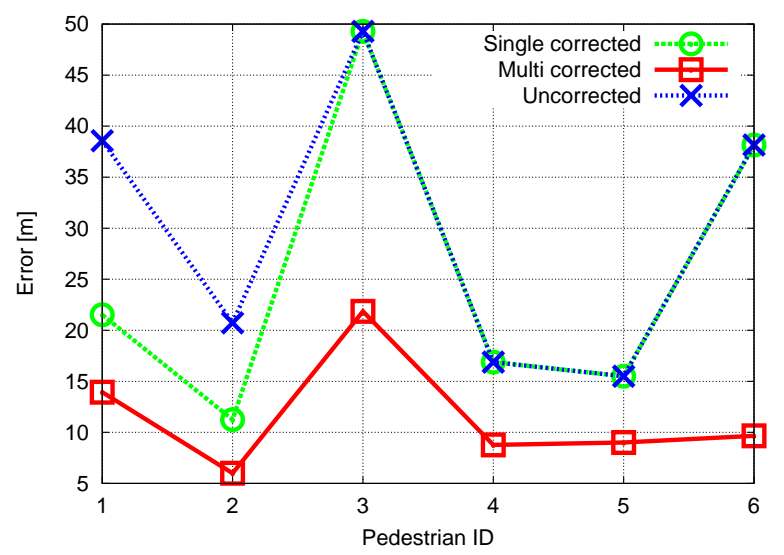

Fig. 2. Average Cartesian error of estimated RFID locations by each pedestrian during the semi-indoor experiment.

\section{B. Outdoor Experiment}

The outdoor experiment was carried out in the city center of Freiburg, a larger urban area containing mainly residential buildings with up to six floors. Due to the multipath propagation problem in this environment, e.g. building structures that reflect satellite signals, recorded GPS tracks were manually corrected for gaining acceptable ground truth data. Figure 3 (a) depicts the recorded PDR trajectories in the same way as described for the semi-indoor experiment. During this experiment, the pedestrians walked for approximately 9000 meters, while deploying in total 39 RFID tags. Figure 3 (b) shows the merged RFID graph after the correction and Figure 3 (c) the ground truth data. As can be seen from (b) and (c), the corrected graph is close to ground truth. Figure 4 shows the average Cartesian error of estimated RFID locations computed in the same way as previously described. As can be also seen here, the correction based on the unified graph yields better results for each single pedestrian. Note that here the correcting of single pedestrian tracks without data sharing leads to no significant improvements due to the lacking of loops on their own trajectories.

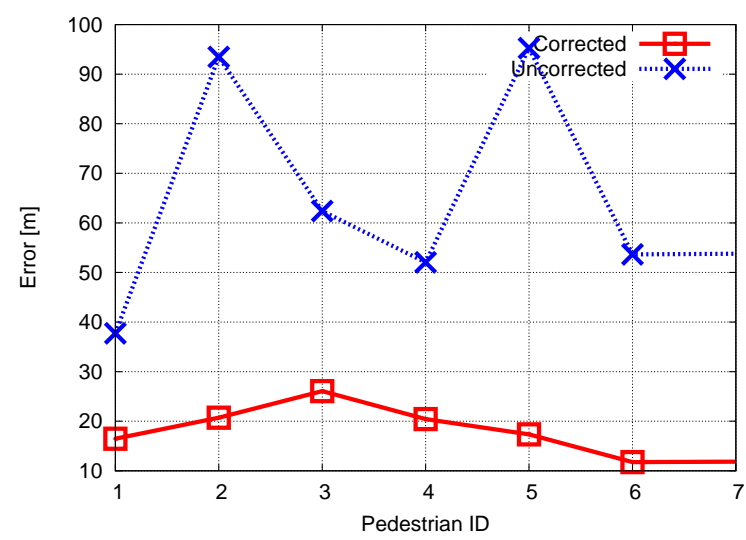

Fig. 4. Average Cartesian error of estimated RFID locations by each pedestrian during the outdoor experiment.

\section{Decentralized SLAM Experiment}

The last experiment was conducted in order to evaluate the decentralized execution of RFID-SLAM. This was carried out by simulating the step-wise execution of trajectories, i.e. one pedestrian after another, on the map. For this purpose the trajectories recorded during the outdoor experiment were used.

The simulation was carried out in the following way: After the execution of an agent's trajectory the subgraphs $R_{i}$ of all RFIDs passed by the agent and the resulting subgraph $A_{i}$ learned by the agent when reaching its goal, were computed. Furthermore, the computed subgraph $R_{i}$ were stored for the execution of the next pedestrian in the sequence. Then, the optimized RFID graph was computed from $A_{i}$ for determining the track error with respect to ground truth. Note that the $R_{i}$ did not necessarily consist of completed trajectories, but of sub-trajectories from preceding agents ending at RFID $i$. Consequently, the emergence of loops depends on the sequence of executing single trajectories. Therefore, all 720 possible sequences from six agents were simulated while averaging their results. Figure 5 depicts the mean Cartesian error and variance of the trajectories walked by the first, second, third, ..., sixth pedestrian in the sequence. As can be seen from the figure, the more pedestrians traveled through the environment, the better the pose estimate of a single pedestrian can be improved. Hence, the last pedestrian was able to achieve a nearly two times higher pose accuracy than the first one by closing loops that were constructed by joining subgraphs from other pedestrians.

\section{CONCLUSIONS AND FUTURE WORKS}

We introduced a novel method for distributed SLAM by exploiting the advantages of RFID technology. The result shows that sharing information between single pedestrians facilitates the global optimization of their 


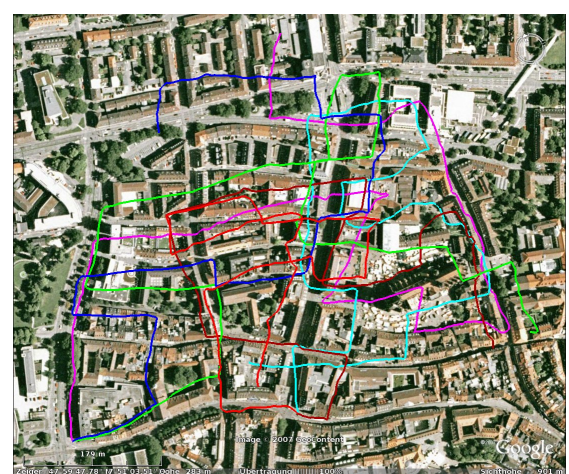

(a)

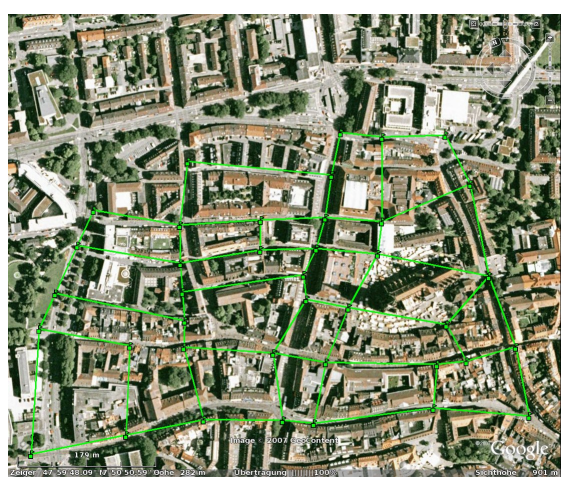

(b)

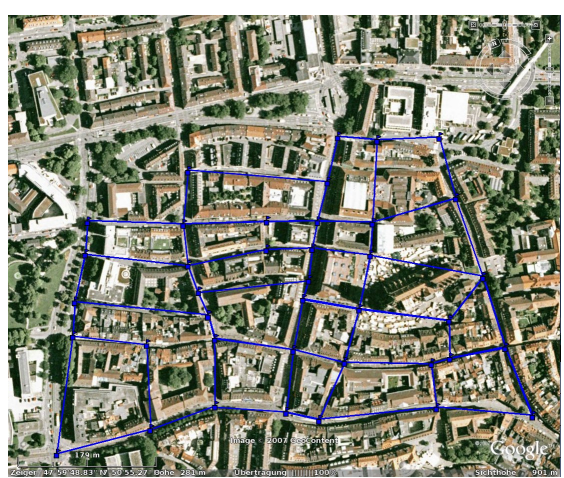

(c)

Fig. 3. Correcting pedestrian trajectories in an urban environment (the City of Freiburg): (a) Pedestrian trajectories, where different colors indicates the individual pose tracking of each pedestrian. (b) The corrected RFID graph. (c) Manually improved GPS ground truth.

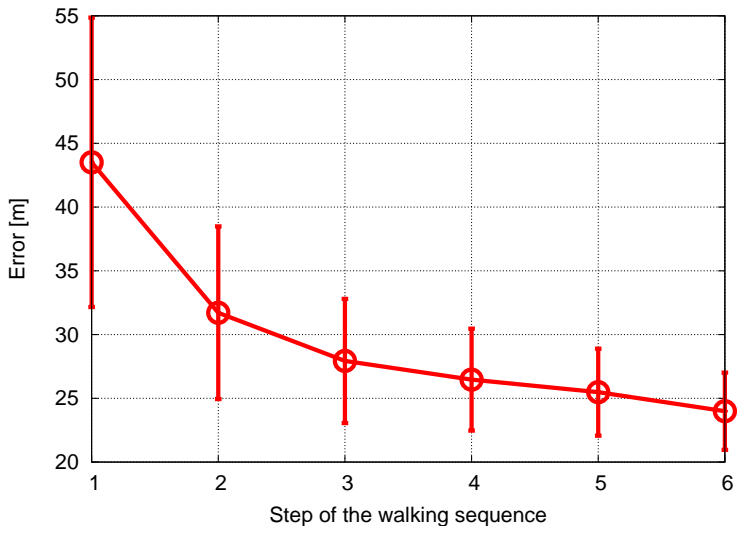

Fig. 5. Result from decentralized SLAM: Average Cartesian pose error of the i-th pedestrian exploiting graph structures for SLAM left by its predecessors within the memory of RFID nodes. Averages are computed from all possible 720 sequences of the six pedestrians.

individual paths, even if they are not able to communicate directly. Obviously, the process of data sharing can further be accelerated if subgraphs are additionally exchanged via radio communication. Moreover, the introduced method allows to apply SLAM without requiring pedestrians to intentionally loop, which is a necessary requirement if applying SLAM in the real-world, particularly when humans are involved.

In future work we will investigate the approach in purely indoor environments while utilizing real RFIDs. Furthermore, we will aim on reducing the number of required RFIDs by utilizing active transponders that can be detected within distances of more than 10 meters, while estimating their distance from signal strength. Finally, it is planned to evaluate the system with data captured by a team of firefighters during an emergency response mission exercise.

\section{REFERENCES}

[1] J. Bohn and F. Mattern. Super-distributed RFID tag infrastructures. In Proc. of the 2nd European Symp. on Ambient Intelligence, number 3295, pages 1-12. Springer, 2004.
[2] R. Fachberger, G. Bruckner, L. Reindl, and R. Hauser. Tagging of metallic objects in harsh environments. In Sensoren und Messsysteme, University of Freiburg, 2006.

[3] Eric Foxlin. Pedestrian tracking with shoe-mounted inertial sensors. IEEE Comput. Graph. Appl., 25(6):38-46, 2005.

[4] A. Kealy G. Retscher. Ubiquitous positioning technologies for modern intelligent navigation systems. The Journal of Navigation, 59:91-103, 2006.

[5] M.S. Grewal, L. R. Weill, and A. P. Andrews. Global Positioning Systems, Inertial Navigation, and Integration. John Wiley \& Sons, 2001.

[6] D. C. T. Judd. A personal dead reckoning module. In Proc. of the Inst. of Navigation's GPS Conf., pages 169 - 170, 1997.

[7] G. Kantor, S. Singh, R. Peterson, D. Rus, A. Das, V. Kumar, G. Pereira, and J. Spletzer. Distributed search and rescue with robot and sensor team. In Proc. of the Fourth Int. Conf. on Field and Service Robotics, pages 327-332. Sage, 2003.

[8] J. Kim, S. Ong, E. Nettleton, and S. Sukkarieh. Decentralised approach to unmanned aerial vehicle navigation: without the use of GPS and preloaded map. Journal of Aerospace Engineering, (6):399-416, 2004.

[9] A. Kleiner, N. Behrens, and H. Kenn. Wearable computing meets multiagent systems: A real-world interface for the RoboCupRescue simulation platform. In First International Workshop on Agent Technology for Disaster Management at AAMAS06, Hakodate, Japan, 2006.

[10] A. Kleiner, J. Prediger, and B. Nebel. RFID technology-based exploration and SLAM for search and rescue. In Proc. of the IEEE/RSJ Int. Conf. on Intelligent Robots and Systems (IROS), Beijing, China, 2006.

[11] Q. Ladetto and B. Merminod. In step with INS: Navigation for the blind, tracking emergency crews. GPSWorld, pages 30-38, 2002.

[12] Q. Ladetto, B. Merminod, P. Terrirt, and Y. Schutz. On foot navigation: When GPS alone is not enough. Journal of Navigation, 53(02):279-285, 2000.

[13] W. Lechner, S. Baumann, and K. Legat. Pedestrian navigation - bases for the next mass market in mobile positioning. In Proc. of Locellus 2001, pages 79-90, 2001.

[14] F. Lu and E. Milios. Globally consistent range scan alignment for environment mapping. Auton. Robots, 4:333-349, 1997.

[15] MicroSensys. Homepage. http://www.microsensys.de/, 2007.

[16] L.E. Miller, P.F. Wilson, N.P. Bryner, J. R. Guerrieri, D. W. Stroup, and L. Klein-Berndt. RFID-assisted indoor localization and communication for first responders. In Proc. of the Int. Symposium on Advanced Radio Technologies, 2006.

[17] E. Nettleton, S. Thrun, and H. Durrant-Whyte. Decentralised SLAM with low-bandwidth communication for teams of airborne vehicles. In In Proc. of the Int. Conf. on Field and Service Robotics, Lake Yamanaka, Japan, 2003.

[18] S. Reece and S. Roberts. Robust, low-bandwidth, multivehicle mapping. In Eighth IEEE Int. Conf. on Information Fusion, Philadelphia, Penn., 2005. 